\title{
Intelligent legged climbing service robot for remote maintenance applications in hazardous environments
}

\author{
Bing L. Luk ${ }^{\mathrm{a}, *}$, David S. Cooke ${ }^{\mathrm{b}}$, Stuart Galt ${ }^{\mathrm{c}}$, Arthur A. Collie ${ }^{\mathrm{d}}$, Sheng Chen ${ }^{\mathrm{e}}$ \\ ${ }^{a}$ Department of Manufacturing Engineering and Engineering Management (MEEM), City University of Hong Kong, \\ Tat Chee Avenue, Kowloon, Hong Kong \\ ${ }^{\mathrm{b}}$ BOC Edwards, HSM, York Road, Burgess Hill, West Sussex, UK \\ ${ }^{\mathrm{c}}$ Almos Systems, 7 Mandora Bend, Carramar, WA 6031, Australia \\ ${ }^{\mathrm{d}}$ Climbing Robot Company Ltd., 58 a Rails Lane, Hayling Island, Hants, UK \\ e Department of Electronics and Computer Science, University of Southampton, Southampton, UK
}

Received 24 January 2003; received in revised form 5 June 2005; accepted 29 June 2005

\begin{abstract}
Wheeled-tracked vehicles are mainly suitable for relatively flat terrain. Legged-vehicles, on the other hand, have the potential to handle wide variety of terrain. Robug IIs is a legged climbing robot designed to work in relatively unstructured and rough terrain. It has the capability of walking, climbing vertical surfaces and performing autonomous floor to wall transfer. The sensing technique used in Robug IIs is mainly tactile and ultrasonic sensing. A set of reflexive rules has been developed for the robot to react to the uncertainty of the working environment. The robot also has the intelligence to seek and verify its own foot-holds. (C) 2005 Elsevier B.V. All rights reserved.
\end{abstract}

Keywords: Legged robot; Climbing service robot; Insect inspired robot; Pneumatic control; Fuzzy logic

\section{Introduction}

Large buildings and tall structures often require regular inspection and maintenance in order to ensure the integrity of their construction. Failing to carry out proper maintenance could cause life or damages to property, which could sometimes result in crimi-

\footnotetext{
* Corresponding author. Tel.: +852 2788 8673; fax: +85227888017 .

E-mail address: meblluk@cityu.edu.hk (B.L. Luk).
}

nal charges. The traditional manual method normally requires the installation of costly scaffoldings or gondolas. Human workers must stand on these scaffoldings or gondolas in mid-air and at high altitude to carry out inspections and repairs. Inevitably, this is a slow and costly maintenance process. Also, in some industries, such as off-shore, nuclear, chemical and power generation industries, inspection by human workers is hazardous and difficult if not impossible. In view of this difficulty, a number of climbing robots have been developed to carry out glass curtain wall cleaning [1-3], 
bridge inspections [4], ship hull inspections [5,6], and painting of large buildings [7]. However, based on experiences of some previous research projects [8-10] in applying climbing robots for remote maintenance applications, it was found that many buildings such as nuclear reactor pressure vessels had very limited access. As a result, a great deal of effort had to be made in order to launch the robots onto the buildings or structures to carry out the required inspection work. This will inevitably slow down the maintenance process and increase the risk of workers who are responsible for installing the robots. A mobile robot which has the capability of walking through a service entrance and then transferring itself onto the vertical surface of the inspected building can save time and reduce risk to human workers who may have to launch the mobile robot in difficult circumstances.

Ninja-I [11,12], a quadruped wall climbing robot, was developed with the capabilities of floor-to-wall transfer. However, its legs were not articulated. As a result, it was not easy to keep the centre of gravity of the robot close to the surface and hence could bring on excessive bending moment on the foot during climbing and transferring between horizontal and vertical surfaces. In addition, the leg structure restricted its reachable range. Ninja-II [13] overcame the aforementioned problems by introducing articulated structure to its legs. However, the robot did not have any gripper on the underside of its body for sitting on the surface during remote operation and hence it did not provide a stable platform for some maintenance operation such as metal cutting which could induce large reaction force to the robot. Besides Ninja series of robot, ROBIN [14], a biped-climbing robot, has attempted to tackle the floor-to-wall transition but no results were shown. Another biped robot, RAMR1 [15-18], was developed by Michigan State University for reconnaissance operations in urban environment. The robot was capable of transferring between orthogonal surfaces. In order to make the robot to work in confined space, RAMR2 [18-20] was developed with its revolute hip joint replaced by a prismatic joint. To increase the reachable range without sacrificing the ability to work in confined space, a new version of RAMR robot was being designed by the University of Utah [21]. The new design provided a hybrid hip joint with discrete prismatic and revolute motion. Despite that these biped climbing robots could perform orthogonal plane transfer, their climbing mechanism was not very reliable since only one foot was gripping on the surface at any one time when the robot was climbing; a large turning moment could be induced on the gripping foot when the free leg stretches sideways during climbing and this large turning moment could cause the gripping foot to detach from the surface [11]. In addition, the biped structure in general does not provide a stable platform for installing tool package and carrying out maintenance work.

Although a lot of research works has been conducted to develop suitable mechanical mechanisms for climbing, very few have addressed the issue of searching suitable footholds for gripping. For a climbing robot, the ability of finding its own footholds on wall surfaces is essential for remote maintenance applications since the surface condition for this type of applications is generally poor. Ninja robots had partially addressed this issue by developing a "Valve-regulated Multiple" (VM) sucker [11]. The VM sucker was partitioned into a number of smaller chambers and the air flow of each chamber was controlled by a passive mechanical valve. This passive valve would be shut off automatically by excessive air flow caused by air leakage through cracks or any imperfection on the surface. This mechanism could handle very small irregularities and grooves on the wall surface but was not capable of handling large surface defects. For large surface defects, it is necessary for the robot to search for its own suitable footholds.

Robug IIs was designed to address the above problems. It had adopted the articulated leg structure with a thoraxial joint to achieve large reachable range and at the same time to keep its body close to the surface to reduce excessive bending moment. It had the ability to walk from the floor to the wall and was also capable of climbing over obstacles and had the intelligence to seek and verify foot-holds. This allowed the robot to work in relatively unstructured environments. In order to provide a stable platform for installing tool package and carrying maintenance tasks, vacuum grippers were attached to the underside of the body in order to allow the robot to sit on the surface during maintenance operation.

This paper will first give a brief description of Robug IIs' system. The rest of the paper will concentrate on describing the control method, the movements and the behaviours used by Robug IIs for wall climbing, climbing over obstacles and floor to wall transfer. 


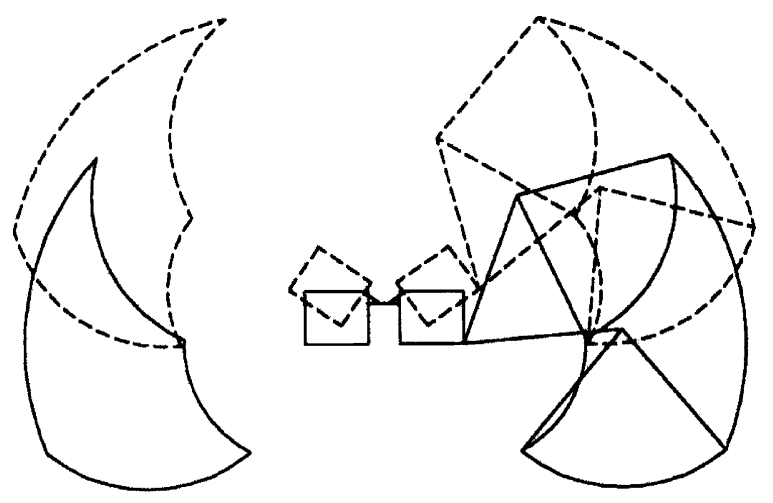

Fig. 1. Robug IIs workspace.

\section{Mechanical system}

Robug IIs was a four limb articulated leg robot powered by double acting pneumatic cylinders. Pneumatic actuation is particularly suitable for climbing robots, due to its high force to weight ratio and inherent compliance [25], which allowed an elegant terrain adapting motion. The robot adopted an endoskeletal structure; an internal frame was used to provide the required strength and stiffness for locomotion as well as locations for the joints, whilst the external actuators acted as the prime mover. The advantage of using this structure is that it is more practical for fabrication and maintenance.

The robot consisted of two similar modules. Each module had two mechanical legs and each had a vacuum gripper foot for climbing vertical surfaces. The robot also had three vacuum suckers on its underside. The two modules were joined by a pivot to form a thoraxial joint with a pneumatic cylinder to bend the body. The arrangement increased the effective moving angles of the legs and reduced the stress exerted on the legjoints (see Fig. 1). This gave the robot the amount of flexibility required for the floor-to-wall transfer.

Each leg had three degrees of freedom and was organised as a spider-like structure (see Figs. 2 and 3). This leg mechanism provided the ability to negotiate and climb over obstacles. It also had the advantage of keeping the body close to the surface, which increased the stability of the robot. An open three bar linkage mechanical structure is used to provide all three degrees of movement of each leg. The anchorage points of the hip and abductor cylinders, and the hip joint were widely separated to reduce stresses on the chassis.

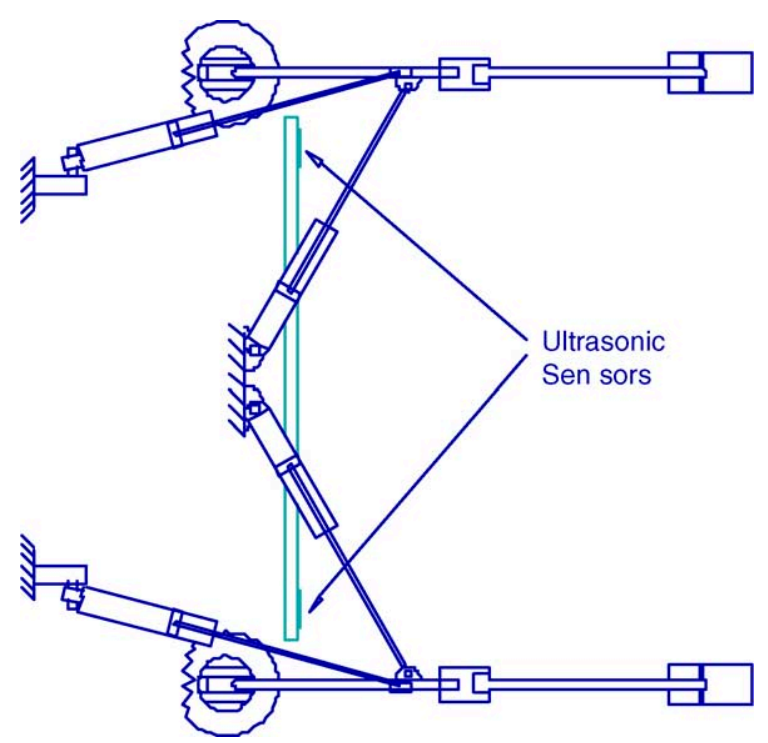

Fig. 2. Plan View of the structure of the two front legs.

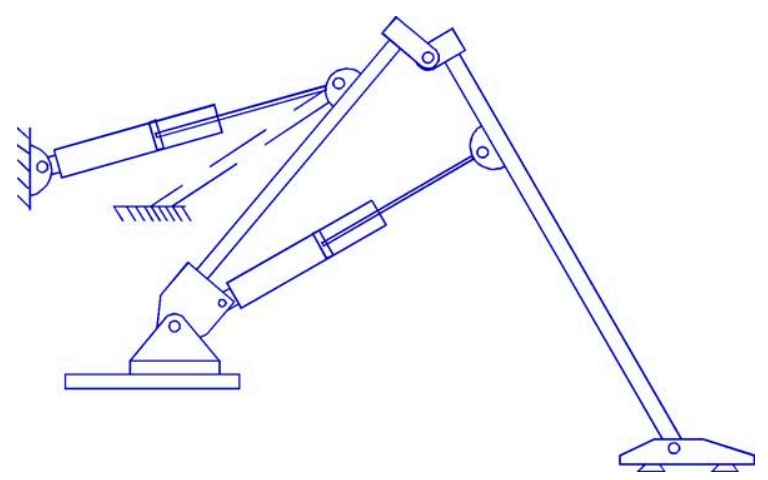

Fig. 3. Elevation view of the leg structure.

The gripper foot was attached to the leg by a ball joint, this provided the gripper foot with the flexibility required to align itself with uneven surfaces. These gripper feet and the base suckers were driven by compressed-air ejector pump and could provide a pull-off force corresponding to $80 \%$ atmospheric pressure.

\section{Control system}

Each leg was controlled by an on-board dedicated microcontroller board. These controller boards provided position, force and compliant control modes. An 


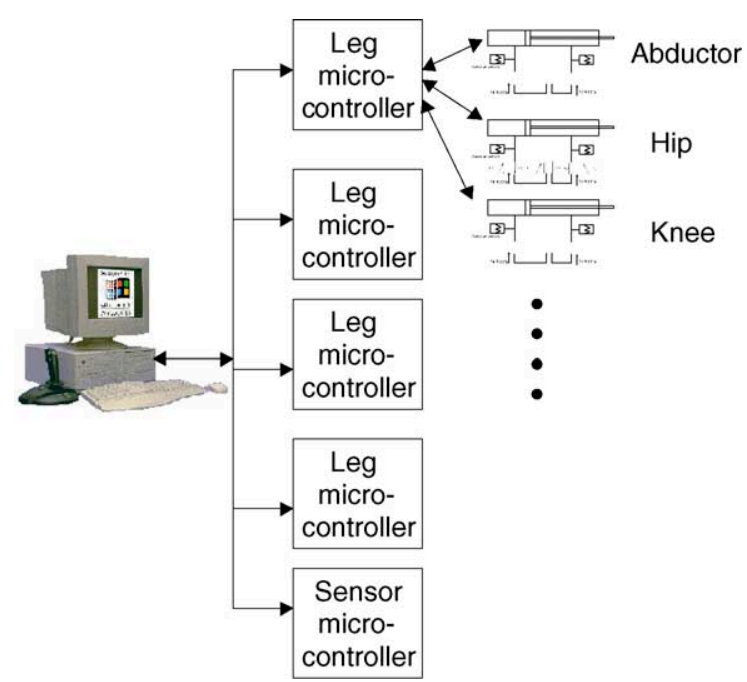

Fig. 4. Computer control system.

additional board was used to provide sensor information from the robot. A supervisory computer at the user end provided the human-machine interface, path planning and functions necessary for co-ordinated motion. A master-slave configuration (see Fig. 4) via a serial link was used to network the supervisory computer and all the leg controllers. A specially designed protocol was used for passing commands and information between the Supervisory computer and the robot. All the important commands were arranged as absolute commands. The repetition of the same command did not affect the final movement.

Each pneumatic cylinder was controlled by three electrically controlled valves, which can allow air to be

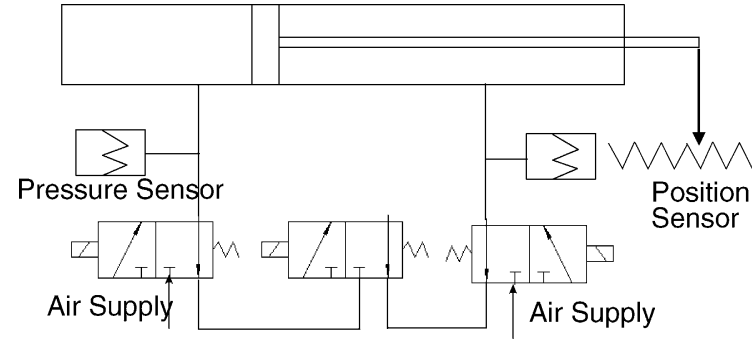

Fig. 5. The valve arrangement for cylinder control.

in both sides of the piston simultaneously (see Fig. 5). Pulse width modulation method was used to drive the cylinder. Since pneumatic action system is non-linear in nature [22,23], a fuzzy logic controller (FLC) has been developed to improve its performance. The fuzzy controller consisted of four primary components, a control rule base, a decision-making inference engine and fuzzification and defuzzification interfaces. The structure of the fuzzy controller is illustrated in Fig. 6. The fuzzification interface quantised the system input variables onto approximate linguistic measures defined by fuzzy sets having the membership functions shown in Fig. 7. The system variables to be observed were defined as $e$ (cylinder position error) and $\Delta e$ (cylinder position error difference)

$e(k) \triangleq p_{\mathrm{T}}(k)-p_{\mathrm{A}}(k)$

$\Delta e(k) \triangleq e_{0}(k)-e_{0}(k-1)$

where $p_{\mathrm{T}}(k)$ was the target cylinder position and $p_{\mathrm{A}}(k)$ was the measured cylinder position at the $k$ th sampling

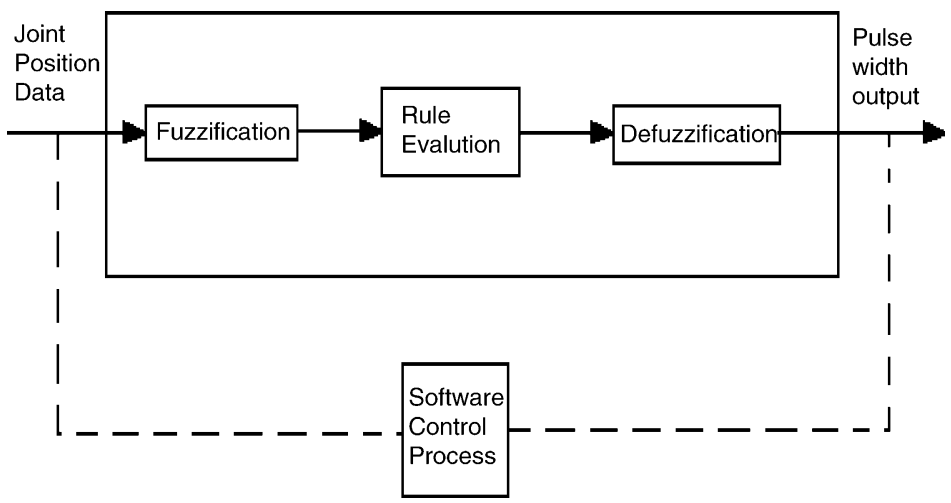

Fig. 6. Structure of the fuzzy controller. 


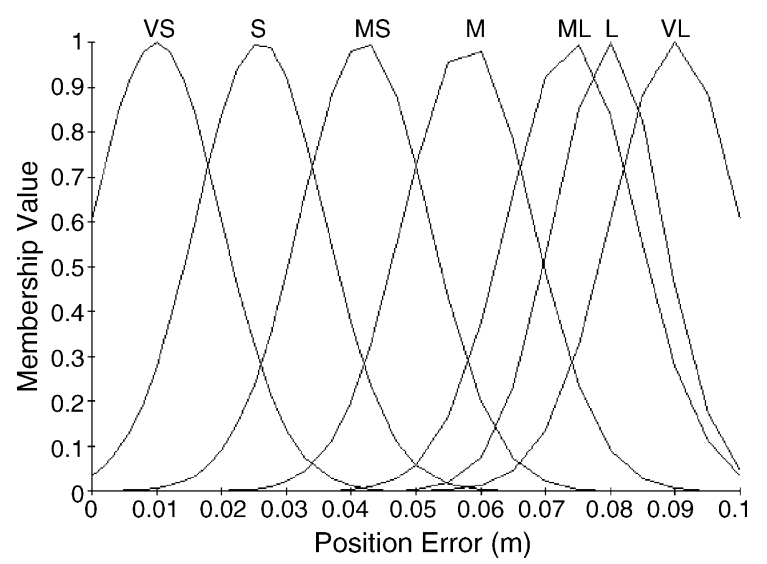

Fig. 7. Fuzzy error sets.

instant.The following linguistic variables were used as labels of the fuzzy sets: VS, Very Small; S, Small; MS, Medium Small; M, Medium; ML, Medium Large; L, Large; VL, Very Large.

The fuzzy rule set was designed to manage the markspace ratio in a pulse width modulation control algorithm according to the position error and position error difference. The control rules are defined in a knowledge base and are represented in linguistic form in Table 1, which has $e$ (cylinder position error) down the left hand column and $\Delta e$ (cylinder position error difference) along the top row. The first element in Table 1 states 'IF /position error) is Very Small AND /change in position error) is Very Small THEN 〈pulse width〉 is Very Small. The control rules are processed within the fuzzy inference engine, which generates a resultant output fuzzy set.

The defuzzification interface used the centre-of-area method to compute a singleton control value for imple-

Table 1

The fuzzy PD rule table

\begin{tabular}{llllllll}
\hline$e_{0}$ & $\Delta e_{0}$ & & & & & & \\
\cline { 2 - 7 } & VS & S & MS & M & ML & L & VL \\
\hline VS & VS & VS & VS & VS & S & MS & M \\
S & VS & S & S & S & MS & M & ML \\
MS & VS & S & S & MS & M & ML & L \\
M & VS & S & MS & M & ML & L & VL \\
ML & NM & MS & M & ML & L & L & VL \\
L & MS & M & ML & L & L & L & VL \\
VL & M & ML & L & VL & VL & VL & VL \\
\hline
\end{tabular}

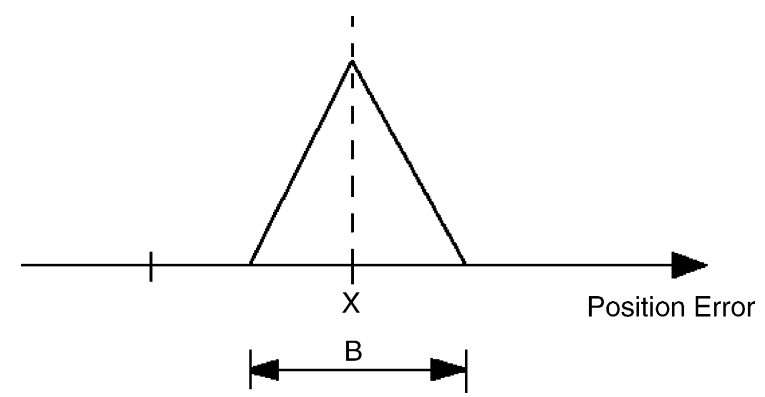

Fig. 8. Fuzzy set shape definition variables.

mentation from the output fuzzy set; this method was used as it produces a gradually changing control output.

Although it is simple to program a fuzzy system using simple rules and membership functions, the tuning of the system performance is a largely subjective process and as a result the design of fuzzy logic controllers is mostly a trial-and-error procedure with extensive tuning of the relational. However, it is possible to automatically improve the performance of the system by using genetically inspired learning strategies now commonly referred to as Genetic Algorithms (GAs) [24]. GAs are particularly good for optimising fuzzy sets as they can explore a search space in many directions simultaneously while avoiding local minima.

In coding the Genetic Algorithm, there must be some defined protocols that enable the GA to understand information regarding the fitness function that is being optimised. Consideration must be given to both the encoding of the membership functions within the GA and the methods by which the GA controls the shape of the fuzzy sets.

The membership functions of the fuzzy sets used were represented by two coefficients defining the function peak position $(x)$ and size of the base (B) (see Fig. 8) then the GA only needed to be coded with these two parameters in order to make adjustments to the fuzzy sets. Therefore the 'chromosomes' of the GA only represented the disparity between the proposed fuzzy sets and the fuzzy sets from the previous generation. For the first generation, adjustments were made at random on the initial fuzzy sets. The following generations evolved a resultant fuzzy set from the previous generation, which had been selected by weighted-random analysis of the fitness levels.

The factors to be considered in evaluating the population fitness levels were the time to reach target posi- 


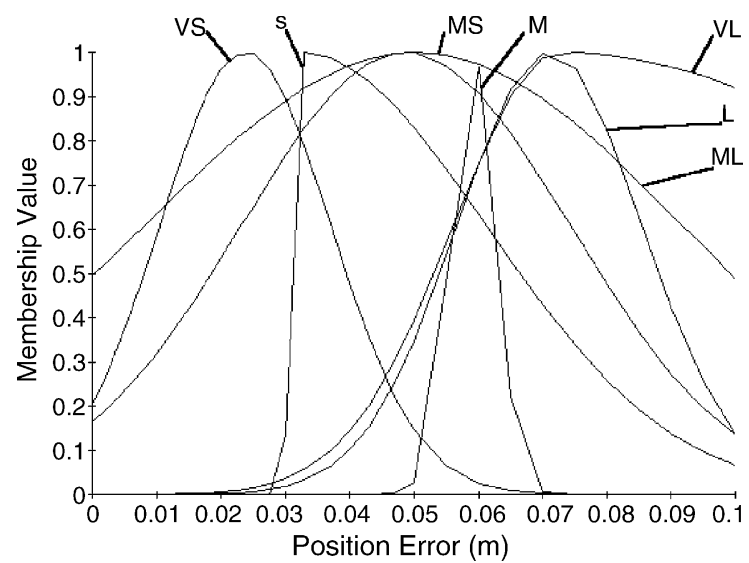

Fig. 9. The membership functions after GA optimisation.

tion, the settling time and the precision required. In the event of the system exhibiting such qualities as slow response time or excessive overshoots the chromosome fitness value could be reduced, lowering the probability of that chromosome reproducing and evolving the attributes into the next generation. This method tailors the learning process to eliminate characteristics that are not permitted to exist within the system.

For tuning the fuzzy controller, a population size of 50 classifiers was used, each 32 bits long ( 4 bits for each fuzzy set). The GA was allowed to run for 100 generations. These parameters were found to be adequate through experience of using the system. The resulting GA-generated optimal fuzzy membership functions are shown in Fig. 9. The response of the fuzzy PD controller is displayed in Fig. 10. As compared with the perfor-

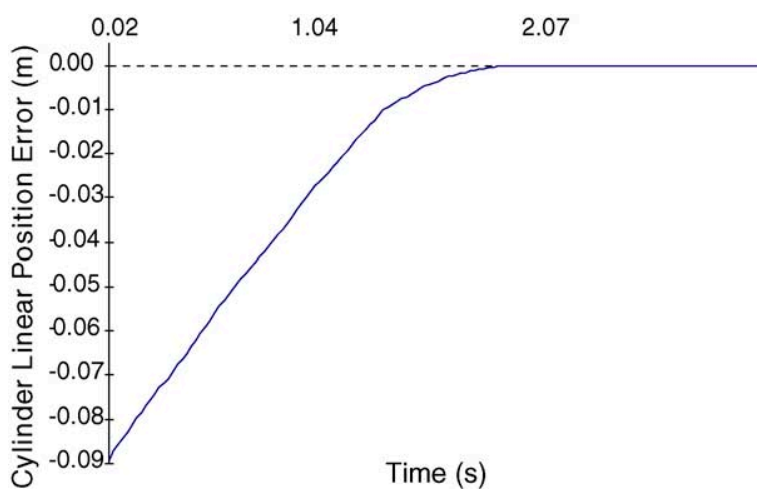

Fig. 10. Cylinder response with Fuzzy Logic PD controller.

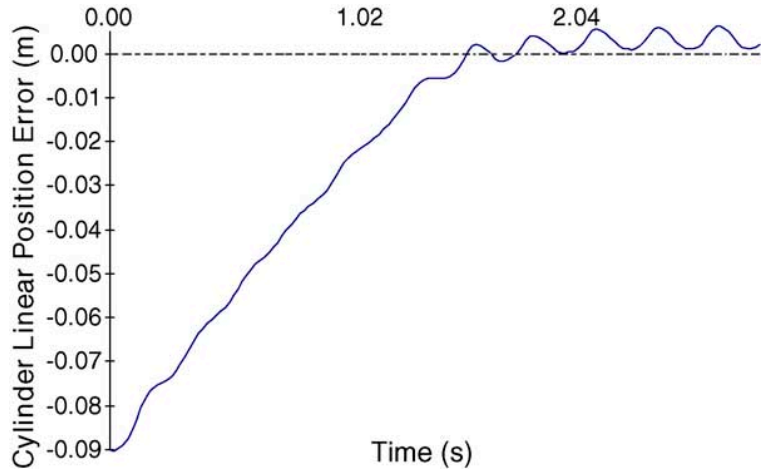

Fig. 11. Cylinder response with PD controller.

mance of a standard PD controller (see Fig. 11), the fuzzy controller can increase the accuracy of the system to $+/-0.3 \mathrm{~mm}(0.2 \%$ of the rod extension range). In addition, the small oscillations occurred in the standard PD control method has also been eliminated (see Fig. 11).

Although Robug was mainly a sensory-based robot and used its reflexive behaviours (see Section 4 for detail) to handle unstructured environments, the proposed fuzzy control method with GA tuning did help to improve its walking and climbing performance. Firstly, it provided a smooth control for the legs and body movements since it could eliminate the small oscillations occurred in the standard PD control method. Secondly, with the improved position accuracy, it enabled the robot to make the maximum strike for each of its walking or climbing steps. Based on authors' experience, the proposed control method could actually reduce $15 \%$ of the time required by the Robug to reach its target location. In addition, as each leg had sufficient strength to carry sensors, the improved accuracy allowed the robot to use its leg as a scanning probe to look for structure defects.

\section{Behaviours}

Robug IIs was designed to work in relatively unstructured environment. In order to accommodate the uncertainty in the working environment, Robug IIs was designed as a sensory-based control robot rather than a pure position control robot. The sensing technique used in Robug IIs was mainly tactile sensing. 


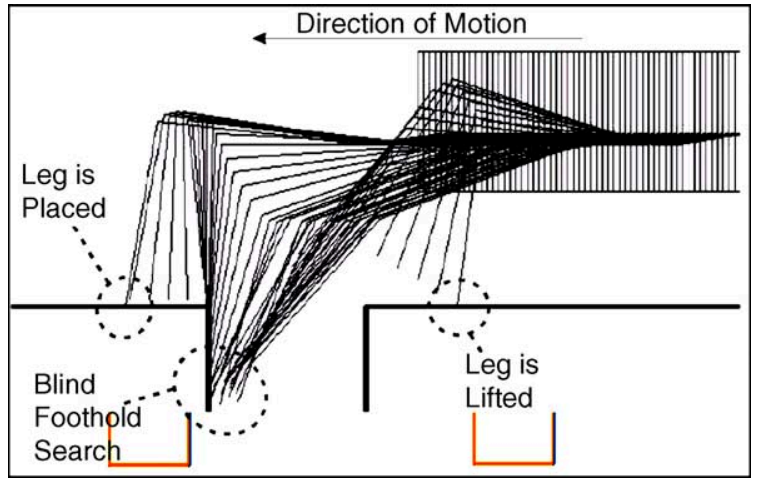

Fig. 12. Blind foothold search and obstacle avoidance.

Each leg was equipped with pressure sensors and potentiometers to provide force and position information of the leg. The leg could be used to feel for obstacles and the level of the surface. The compliant control of the leg allowed the robot to perform tactile sensing without damaging the building structure. The vacuum sensors at the feet and base suckers gave the measurements of gripping force on the surface. Ultrasonic sensors were also installed at the front of the robot for measuring distance of the object.

In order to allow the robot to handle the uncertainty in unstructured environments, a set of rules were developed to define the behaviours of the robot for reacting to the sensory information fed back from the environment.

These rules are listed as follow:

1. No leg will move unless the robot has at least three suckers gripping firmly on the surface. This rule prevents the robot from falling when it is climbing a wall.

2. A reflex action for searching foothold is build into the leg controller (see Fig. 12). This arrangement provides a fast searching movement. The reflex action allows the leg to search for a foot-hold in front. It also provides an oscillating movement, which can be used to remove loose material on the surface. This facility will increase the chance of finding gripping surface.

3. No two legs will be allowed to apply force in the opposite directions. This prevents any damage to the chassis caused by excessive force since each cylinder can exert $500 \mathrm{~N}$ force.
4. Always use minimum force for searching foothold and negotiating object. This rule ensures the robot will not cause any damage to the climbing structure.

5. Since the robot is capable of walking, climbing and floor-to-wall transfer, the gravity force exerted on each joint can vary significantly when the robot is lifting the body. A simple compensation scheme is used to overcome the gravity force where additional pressure can be added gradually by increasing the additional force demand variable in the leg controller.

6. The robot will adjust the position of the legs to spread out the force exerted on each leg.

The above rules form the basic behaviours of the robot. They provide the local autonomy to each limb. Other high level of strategies can be added to provide the task oriented applications.

\section{Walking and climbing movement}

The movement of the robot can be grouped into two main types: (1) normal climbing or walking, (2) climbing over obstacle or floor to wall transfer.

During the normal climbing or walking, the thoraxial actuator will be fully extended so that the two mechanical modules are level with each other. Base suckers are used to assist climbing and walking. In this mode, the movement of the robot can be divided into four basic steps: (1) move the legs to follow a pre-planned trajectory, (2) get the legs to grip onto the surface, (3) move the body to follow a pre-planned trajectory and (4) get the base vacuum sucker to grip onto the surface. When the base suckers are firmly attached on the wall, all four legs can move at the same time for fast operation.

Considering the case when the robot is moving forward, the following movements are implemented:

1. The robot starts at the rest position as shown in Fig. 13a.

2. The robot releases all the gripper feet suckers.

3. All four legs will follow a pre-defined trajectory (see Fig. 13b).

4. Each leg starts searching for a gripping surface until a secure grip is verified. The attitude after gripping on the surface is shown in Fig. 13c. 


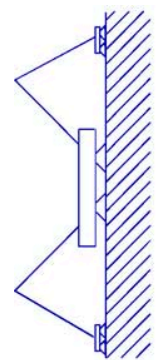

(a)

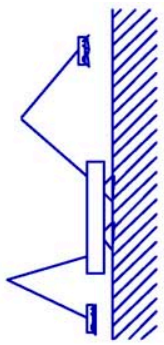

(b)

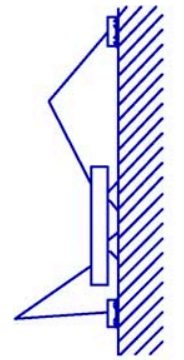

(c)

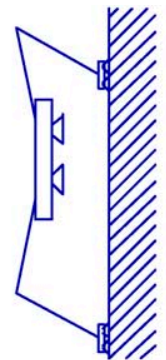

(d)

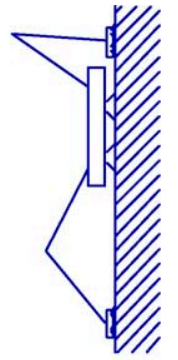

(e)

Fig. 13. Robug IIs wall climbing sequence.

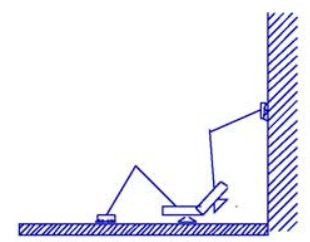

(a)

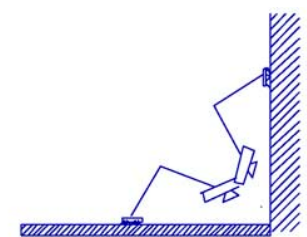

(b)

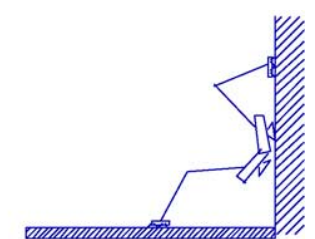

(c)

Fig. 14. Robug IIs floor-to-wall transfer sequence.

5. The robot then releases its belly sucker and levers its body up and follows a pre-planned trajectory as shown in Fig. 13d.

6. The robot starts searching for a gripping surface until a secure grip is verified at the base suckers. The position after the body gripping on the surface is shown in Fig. 13e.

In the case of turning, the front leg on the near side of the turning direction and the rear leg on the off side will move outward. The remaining legs will move inward. The robot will then lift the body and turn to the required direction.

For climbing obstacles or floor-to-wall transfer motions, the robot will use its thoraxial actuator to control the bending angle of the body. This increases the effective angle that the leg can travel and hence enhance the flexibility of the robot for climbing over sharp angled objects or slopes. The base suckers are not normally used in this kind of movement and only one leg is moving at any one time.

When the robot is performing a floor-to-wall transfer operation, a sequence of movements is executed as follow:

1. The robot will walk toward the wall until a full walking pace is not possible. Ultrasonic distance sensors are used to measure the distance. The robot will walk with its front legs spread when it is close to the wall.

2. The robot releases the front feet and front base sucker. It then bends the body up according to slope of the wall.

3. The front legs starts searching for a secure grip on the wall (see Fig. 14a).

4. The robot then releases its rear base sucker and then lift its body up (see Fig. 14b).

5. Each leg will release its gripper feet sucker and search for a secure grip in turn.

6. It lifts its body up and follows a planned trajectory (see Fig. 14c).

7. Step 5 and 6 are repeated until the rear legs are just reaching the floor.

8. The robot will straighten the spine cylinder and the move its body toward the wall until a secure grip is obtained at the base suckers.

9. It then moves its rear legs in a high gait to transfer onto the wall.

\section{Conclusion}

Robug IIs with its insect-like structure and articulated limbs had the mechanical capability to han- 


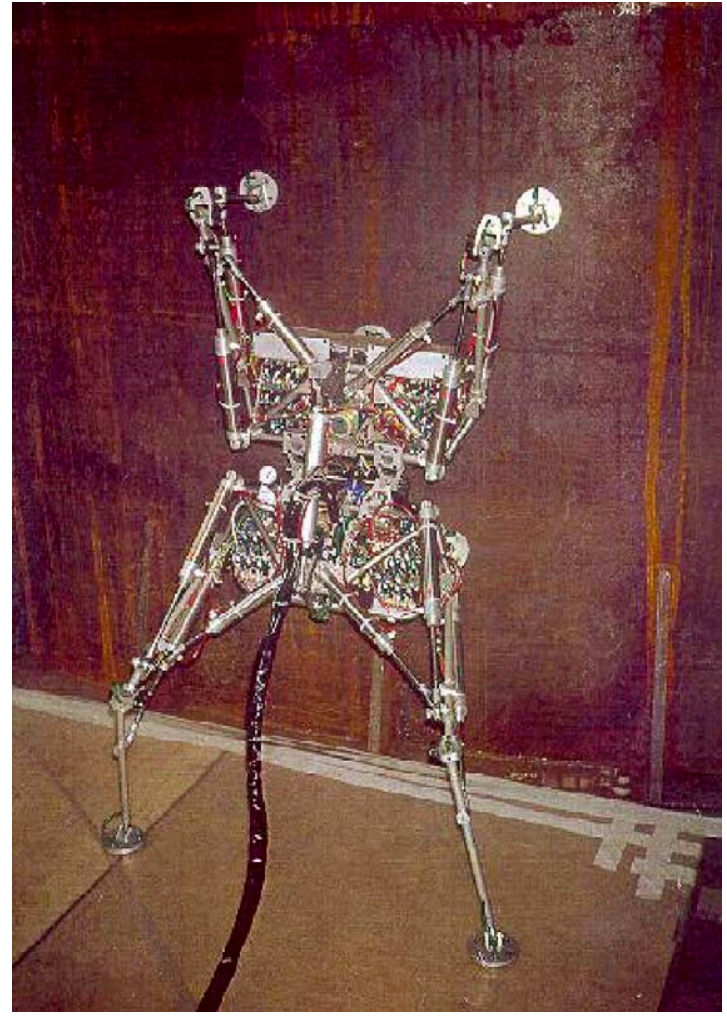

Fig. 15. Robug IIs performing an automatic floor-to-wall transfer.

dle wide variety of the terrain. Pneumatic actuators were used to power the robot in order to reduce the weight of the robot and yet provide enough strength for carrying tools. In order to overcome the deficiency of the standard PID control method in controlling non-linear pneumatic actuators, a Fuzzy control method with GA tuning was developed. The proposed method provided smooth and accurate motions for the robot and hence enhanced the walking and climbing performance. In addition, the improved accuracy also allowed the use of the robot leg as a scanning probe for inspecting structure defects. The reflexive behaviours facilitated the robot to work in relatively unstructured environment. This kind of autonomy had certainly simplified the operation procedures of the robot. The capability of performing automatic floor-to-wall transfer was vital for applications where access to the structure being inspected is limited. The robot was tested on a real-scale model of

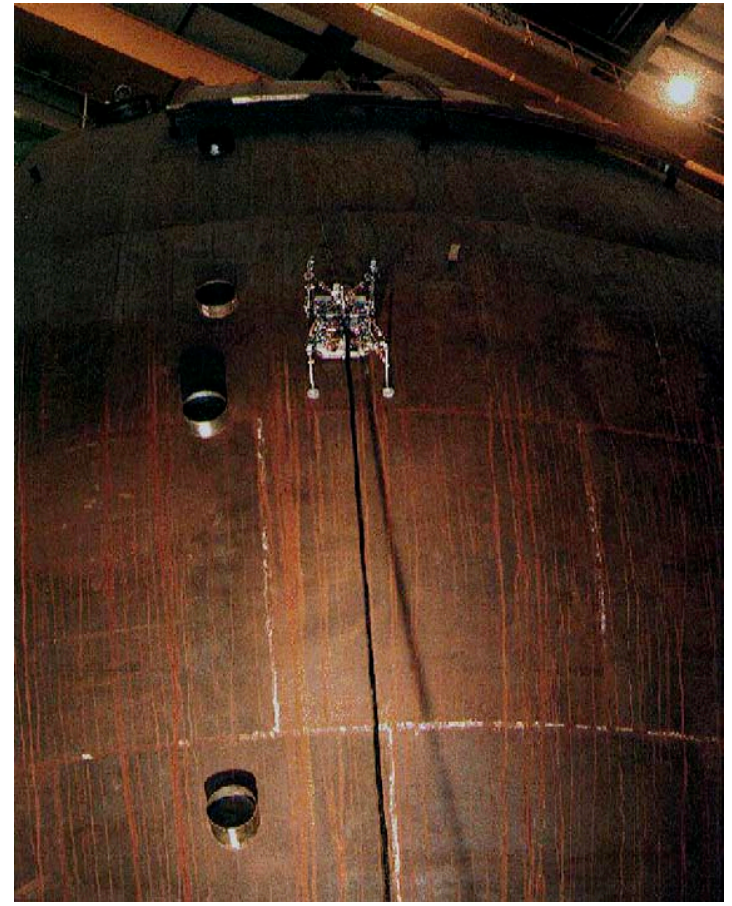

Fig. 16. Robug IIs climbing a Magnox nuclear reactor pressure vessel.

a Magnox nuclear reactor pressure vessel and the result has confirmed the usefulness of the robot (see Figs. 15 and 16).

Further work in navigation system is being carried out to enhance the autonomy of the robot. Since the robot is capable of walking and climbing, the navigation problem is no longer restricted in 2-D space. The robot has to determine whether it should walk round the obstacles or climb over them.

\section{Acknowledgement}

The authors would like to thank Mr. Tim White and Mr. Neal Hewer for their help in developing some of the electronic circuitry. The authors would also like to thank the University of Portsmouth and Magnox Electric/BNFL to facilitate the success of the experimental work. The research work was financially supported by an EPSRC grant (GR/M16894) and Magnox Electric plc. 


\section{References}

[1] S.K. Tso, Y.H. Fung, W.L. Chow, G.H. Zong, R. Liu, Design and implementation of a glass-wall cleaning robot for highrise buildings, in: Proceedings of the World Automation Congress Eighth International Symposium on Robotics with Applications, Maui, Hawaii, June 11-16, 2000 (Paper ID: ISORA123).

[2] S.K. Tso, J. Zhu, B.L. Luk, Prototype design of a light-weight climbing robot capable of continuous motion, in: Proceedings of the Eighth IEEE Conference on Mechatrinics and Machine Vision in Practice, Hong Kong, 27-29 August, 2001, pp. 235-238.

[3] Q.X. Zhang, Z.G. Ren, Z.W. Zhao, Development of a 3W window-cleaning robot for high-rise buildings, in: Proceedings of the Eighth IEEE Conference on Mechatrinics and Machine Vision in Practice, Hong Kong, 27-29 August, 2001, pp. 257-260.

[4] C. Hillenbrand, K. Berns, F. Weise, J. Koehnen, Development of a climbing robot system for non-destructive testing of bridges, in: Proceedings of the Eighth IEEE Conference on Mechatrinics and Machine Vision in Practice, Hong Kong, 27-29 August, 2001, pp. 399-403.

[5] T.P. Sattar, M. Alaoui, S. Chen, B. Bridge, A magnetically adhering wall climbing robot to perform continuous welding of long seams and non-destructively test the welds on the hull of a container ship, in: Proceedings of the Eighth IEEE Conference on Mechatrinics and Machine Vision in Practice, Hong Kong, 27-29 August, 2001, pp. 408-414.

[6] B. Bahr, Y. Yin, Wall climbing robots for aircraft, ship, nuclear power plants, sky scrapers, etc., in: Proceedings of the Fifth International Symposium on Robotics and Manufacturing, Hawaii, USA, August, 1994.

[7] Y. Wang, H. Shao, Wall climbing robot for cleaning and painting, in: Proceedings of the Second International Conference on Climbing and Walking Robots, Portsmouth, UK, September, 1999.

[8] B.L. Luk, T.S. White, D.S. Cooke, N.D. Hewer, The 'Sadie' (sizewell a duct inspection equipment) teleoperated walking climbing robot, in: C. Ealing, T. Schilling (Eds.), Successful Tele-robotic Applications, IMechE, Professional Engineering Publishing Ltd., London and Bury St. Edmunds, UK, 1999, pp. 117-130.

[9] M.S. Burrows, A.A. Collie, A. Curry, Inspection of a nuclear reactor pressure vessel by vacuum attached and magnetically attached mobile robots, in: Proceedings of the Fourth International Symposium on Offshore, Robotics and Artificial Intelligence, Telerobotics In Hostile Environments, Marseille, France, 11-12 December, 1991.

[10] B.L. Luk, A.A. Collie, T.S. White, NERO-A teleoperated wall climbing vehicle for assisting inspection of a nuclear reactor pressure vessel, in: Proceedings of the 13th ASME International Computers in Engineering Conference and Exposition, San Diego, USA, 8-12 August, 1993.

[11] S. Hirose, A. Nagakubo, R. Toyama, Machine that can walk and climb on floors, walls and ceilings, in: Proceedings of the Fifth International Conference on Advanced Robotics, Robots in Unstructured Environments, vol. 1, 19-22 June, 1991, pp. $753-758$.

[12] A. Nagakubo, S. Hirose, Walking and running of the quadruped wall climbing robot, in: Proceedings of the IEEE International Conference on Robotics and Automation, vol. 2, 8-13 May, 1994, pp. 1005-1012.

[13] S. Hirose, K. Kawabe, Ceiling walk of quadruped wall climbing robot NINJA-II, in: Proceedings of the First International Conference on Climbing and Walking Robots (CLAWAR 98), Brussels Belgium, 26-28 November, 1998, pp. 143-147.

[14] R.T. Pack, J.L. Christopher Jr., K. Kawamura, A rubbertuatorbased structure-climbing inspection robot, in: Proceedings of the IEEE International Conference on Robotics and Automation, vol. 3, 20-25 April, 1997, pp. 1869-1874.

[15] M. Yue, M. Minor, N. Xi, R. Mukherjee, Kinematic workspace analyses of a miniature walking robot, in: Proceedings of the IEEE/RSJ International Conference on Intelligent Robots and Systems, vol. 3, 17-21 October, 1999, pp. 1798-1803.

[16] M. Yue, N. Xi, M. Minor, R. Mukherjee, Dynamic workspace analysis and motion planning for a micro biped walking robot, in: Proceedings of the IEEE/RSJ International Conference on Intelligent Robots and Systems, vol. 3, 31 October-5 November, 2000, pp. 1900-1905.

[17] M. Minor, H. Dulimarta, G. Danghi, R. Mukherjee, R. Lal Tummala, D. Aslam, Design, implementation, and evaluation of an under-actuated miniature biped climbing robot, in: Proceedings of the IEEE/RSJ International Conference on Intelligent Robots and Systems, vol. 3, 31 October-5 November, 2000, pp. 1999-2005.

[18] R. Lal Tummala, R. Mukherjee, N. Xi, D. Aslam, H. Dulimarta, J. Xiao, M. Minor, G. Dang, Climbing the walls, in: IEEE Robotics and Automation Magazine, vol. 9, Issue 4, December 2002, pp. 10-19.

[19] J. Xiao, M. Minor, H. Dulimarta, N. Xi, R. Mukherjee, R.L. Tummala, Modeling and control of an under-actuated miniature crawler robot, in: Proceedings of the IEEE/RSJ International Conference on Intelligent Robots and Systems, vol. 3, 29 October-3 November, 2001, pp. 1546-1551.

[20] J. Xiao, N. Xi, J. Xiao, J. Tan, Multi-sensor referenced gait control of a miniature climbing robot, in: Proceedings of the IEEE/RSJ International Conference on Intelligent Robots and Systems, vol. 4, 27-31 October 2003, pp. 3656-3661.

[21] S.P. Krosuri, M.A. Minor, A multifunctional hybrid hip joint for improved adaptability in miniature climbing robots, in: Proceedings of the IEEE International Conference on Robotics and Automation, vol. 1, 14-19 September, 2003, pp. 312-317.

[22] Y. Kawakami, J. Akao, S. Kawai, Some considerations on the dynamic characteristic of pneumatic cylinders, J. Fluid Control 19 (2) (1988) 22-36.

[23] B.W. Andersen, The Analysis and Design of Pneumatic Systems, John Wiley and Sons, USA, 1967 (Library of Congress Catalog Card No. 67-17332).

[24] D.E. Goldberg, Genetic Algorithms in Search, Optimisation and Machine Learning, Addison Welsey, 1989.

[25] A.A. Collie, J. Billingsley, L. Hatley, The development of a pneumatically powered walking robot base, ImechE c377/86 pub, 1986. 


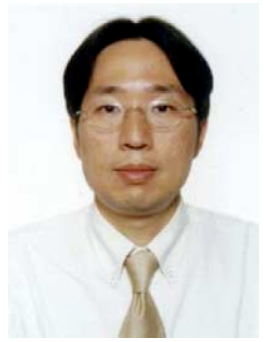

Bing L. Luk received his BSc Degree in Electrical and Electronic Engineering from Portsmouth Polytechnic, UK, in 1985, MSc Degree in Digital Computer Systems from Brunel University, UK, in 1986 and $\mathrm{PhD}$ Degree in Robotics from the University of Portsmouth, UK, in 1991. He joined the Department of Manufacturing Engineering and Engineering Management at City University of Hong Kong in 2000. He previously held research and academic appointments at University of Portsmouth, UK and engineering consultant position at Portsmouth Technology Consultant Ltd. and also other industrial companies. His recent research works include telemedicine research, home automation, non-destructive test methods, machine learning and evolutionary computation methods

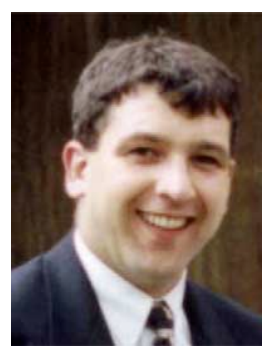

David S. Cooke graduated from Portsmouth Polytechnic with a first class Honours Degree in Mechanical Engineering in 1991. He immediately joined the University of Portsmouth Mobile Robotics Group and worked on enabling robotics projects in collaboration with Portsmouth Technology Consultant Ltd. (Portech) and Nuclear Electric plc (now BNFL Magnox). In 1996 he joined Portech as the Senior Mechanical Engineer taking responsibility for the company's mechanical design function and in a company restructure in 1998 became the new R\&D Manager; taking overall responsibility for the company's robotics work. In 1999 he was awarded a $\mathrm{PhD}$ for his research into "Tractive Mechanisms for Mobile Climbing Robots". In 2001 he joined the Manufacturing Technology Group at BOC Edwards, as a Senior Manufacturing Engineer and is currently enjoying working on a range of projects.

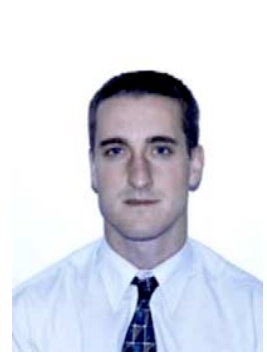

Stuart Galt received his 1st degree for the University of Glasgow, Scotland, in 1994. He then joined the Mobile Robotics Group at the University of Portsmouth, completing his PhD in "Soft Computing Based Control of an Eight-Legged Robot" in 1998. He later joined Portsmouth Technology Consultants (PORTECH). Stuart left the area of robotics research and academia in 2000 to join Raymarine (formerly Raytheon Marine), developing recreational marine electronics. In 2004 he and his family immigrated to Perth, Australia and he is currently employed by Almos Systems, developing a range of meteorological and monitoring products. Contact stuartgalt73@yahoo.com.au

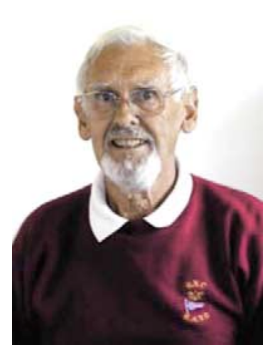

Arthur A. Collie received a BSc in electrical engineering from Glasgow University in 1955 and followed a career in specialising in the development of electromechanical devices. He came to the University of Portsmouth in 1985 from a senior position in high volume manufacturing after being awarded a Royal Society Research Fellow to research legged mobile robots for industrial applications. He headed the Advance Robotics Group in 1988 and was appointed Industrial Professor in 1992. He has many patents and publications in the robotic and other fields and has designed and supplied large climbing robots used in the Nuclear Industry. In 1988 he founded the company, which was to become Portech Ltd. to manufacture and support the industrial robots developed at the university. In1999 he set up the Climbing Robot Company Ltd. to continue the design of vacuum adhesion and magnetic climbing robots.

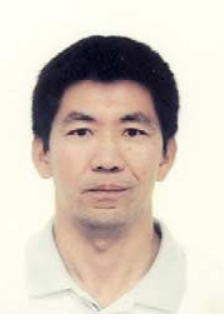

Sheng Chen received the BEng degree in control engineering from the East China Petroleum Institute, Dongying, China, in 1982 and the $\mathrm{PhD}$ degree in control engineering from the City University, London, UK, in 1986. He joined the School of Electronics and Computer Science, University of Southampton, Southampton, UK, in September 1999. He previously held research and academic appointments at the University of Sheffield, Sheffield, UK, the University of Edinburgh, Edinburgh, UK, and University of Portsmouth, Portsmouth, UK. His recent research works include adaptive non-linear signal processing, wireless communications, modeling and identification of non-linear systems, neural networks and machine learning, finite-precision digital controller design, evolutionary computation methods, and optimisation. He has published over 240 research papers. 